\title{
A Novel RFI Suppression Method for Compressive Sensing SAR Imaging
}

\author{
Chaoyun Mai, Ruxin Cui, Jinping Sun \\ School of Electronics and Information Engineering, Beijing \\ Univ. of Aeronautics and Astronautics, \\ Beijing 100191, China \\ maichaoyun@foxmail.com
}

\author{
Bingchen Zhang \\ National Key Lab of MW Imaging Technology, Institute of \\ Electronics, Chinese Academy of Sciences, \\ Beijing 100190, China
}

\begin{abstract}
Synthetic Aperture Radar (SAR) has the all-day, allweather ability to work. It has become one of the important sensors for military reconnaissance and civilian remote sensing. Radio frequency interference (RFI) is the major interference source for low frequency band SAR systems. This paper proposes a novel RFI suppression method for Compressive Sensing SAR. To the compressed sampled echo data, the greedy algorithm is adopted to estimate RFI spectrum with sparse feature, the minimum description length (MDL) criteria is used to estimate the RFI components sparsity. Then to the echo signal of each pulse, the RFI signal components are estimated and filtered in the time domain directly. Lastly, conventional compressive sensing SAR reconstruction algorithm can be applied to achieve imaging output.
\end{abstract}

Index Terms-SAR, RFI suppression, Compressive Sensing, Greedy algorithm, MDL.

\section{INTRODUCTION}

Radio frequency interference (RFI) is the major interference source for low frequency band synthetic aperture radar (SAR) systems. Common RFI include all kinds of radio, television and terrestrial communications signal. Strong RFI signal will seriously affect the quality of SAR imaging. It deteriorates the detection and recognition performance of small target, and even cover real imaging scene ${ }^{[1]}$. How to detect and suppress RFI effectively is an important research direction in data processing of low-band SAR. The existing narrow-band RFI suppressing methods mainly include parameterized methods and non-parameterized methods. Compressive sensing (CS) theory proposed in recent years show that when the signal has sparse features, the serious under-sampled data signal can be used to achieve accurate or approximate reconstruction of signal ${ }^{[2]}$. Since CS technique can effectively reduce the amount of data and save the signal bandwidth, therefore it has been extensive researched and used in the field of SAR imaging ${ }^{[3-6]}$. To the low-band SAR system which uses CS technology, the existence of RFI would undermine the priori condition that with imaging scene scattering coefficient sparsity, making the subsequent imaging process incorrectly. Because the actual RFI unknown, SAR echo signal which contains the RFI components is no longer with sparse features to the sparse base matrix constructed in advance based on the system parameters. At the same time, the existing RFI suppression algorithm based on the Nyquist sampling signal model could not be applied because of compressed sampling is used for echo.

A lot of experiments show that within the spectral range of the SAR signal, the RFI component has obvious sparse feature in the frequency domain. Based on this characteristic, this paper proposes a novel RFI suppression method of CS SAR. To the compressed sampled echo data, the greedy algorithm is adopted to estimate RFI spectrum with sparse features. The minimum description length (MDL) is used to estimate the RFI components' sparsity. Then to the echo signal of each pulse, the RFI signal components are estimated and filtered in the time domain directly. Conventional CS SAR reconstruction algorithm then can be applied to achieve imaging results.

\section{RFI SIGNAL COMPRESSIVE SENSING}

When RFI is existed, SAR echo signal for one pulse can be represented as follows.

$$
y(\tau)=s_{e}(\tau)+s_{r}(\tau)+w(\tau)
$$

Here $s_{e}(\tau)$ is echo of imaging scene, $s_{r}(\tau)$ is RFI signal, $w(\tau)$ is thermal noise of radar system. In the receiver working frequency band, $w(\tau)$ can be viewed as a zero-mean white complex Gaussian noise. For the SAR system of transmitting linear frequency-modulation (LFM) pulse waveform, the power spectrum of the echo $s_{r}$ has a good flatness, so it can be approximated as a white process. According to the existing experimental results, the RFI signal power in the low-band SAR is significantly higher than the scene echo signal. This is mainly because RFI signal is one-way propagation and echo signal is a two-way propagation. At the same time, the spectral range of the actual SAR system has taken the potential RFI suppression into account in the design of the system. And the RFI signal is generally narrowband. So in the spectral range of the SAR signal, RFI component has obvious sparse features.

Set $z(\tau)=s_{e}(\tau)+w(\tau)$, so equation (1) can be changed as follows.

$$
y(\tau)=s_{r}(\tau)+z(\tau)
$$


For RFI suppression, received signal $y(\tau)$ need to be used to detect and estimate RFI signal $s_{r}(\tau)$. But $z(\tau)$ will affect the performance of the detection and estimation, therefore regarded as 'noise' ${ }^{[1]}$.

CS theory proposed in recent years show that if a $N$-length signal $\mathbf{x}$ is sparse on a set of orthogonal basis or transform coefficients of tight frame $\boldsymbol{\Psi}$, a $M \times N(M \square N)$ dimensional observation matrix $\boldsymbol{\Phi}$ independent of transform base can be used to do the linear projection transformation to $\mathbf{x}$, and get the $M$-length observation sample $\mathbf{y}$. Then optimization solving method can be used to reconstruct the original signal $\mathbf{x}$ accurately or high probability from $\mathbf{y}$. That means, to the $N \times N$ dimensional basis matrix $\boldsymbol{\Psi}=\left\{\psi_{1}, \psi_{2}, \cdots, \psi_{N}\right\}$, signal $\mathbf{x}$ can be expressed as follows.

$$
\mathbf{x}=\sum_{i=1}^{N} s_{i} \psi_{i}=\boldsymbol{\Psi} \mathbf{s}
$$

Here $\mathbf{s}$ is $N \times 1$-dimensional column vector constituted from expansion coefficients of $\mathbf{x}$, and $s_{i}=\left\langle\mathbf{x}, \psi_{i}\right\rangle$. If $\mathbf{s}$ has only $K$ nonzero (or large absolute value) coefficients, and other $N-K$ coefficients are all zero (or small absolute value), then the signal is called $K$-sparse. After linear projection transformation of $\mathbf{x}$, Length of $M$ for the observation sample.

$$
\mathbf{y}=\Phi \Psi \mathbf{s}=\mathbf{A s}
$$

Here $\mathbf{A}=\boldsymbol{\Phi} \boldsymbol{\Psi}$ is called CS matrix. $\boldsymbol{\Phi}$ is measurement matrix. The number of observations $M$ is much smaller than the signal length $N$, so linear equations of $\mathbf{x}$ reconstructed directly from the observed data $\mathbf{y}$ is uncertain. CS theory prove that When the matrix $\mathbf{A}$ has restriction isometric properties (RIP), $\mathbf{s}$ can be accurately reconstructed with great probability by observation data $\mathbf{y}$. CS signal reconstruction algorithm is that solving the most sparse solution to meet $\mathbf{y}=\mathbf{A s}$. This problem can be formed as the following constrained optimization problem.

$$
\min \|\mathbf{S}\|_{\ell} \quad \text { s.t. } \quad\|\mathbf{y}-\mathbf{A s}\|_{2} \leq \varepsilon
$$

Here $\varepsilon$ is a noise threshold. Zero norm of $\ell=0$ represents the number of non-zero elements of $\mathbf{s}$, it can be directly used to describe sparse characteristics of the vector to be estimated.

In the CS SAR, compressed samples of the received signal $y(\tau)$ can achieve in different ways. Take the form of time domain non-uniform random sampling as example, that

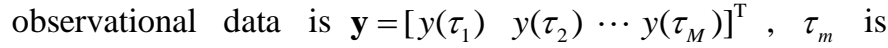
unequal intervals random sampling time. Assumed that conventional sampling frequency $F_{s}$ which meets the Nyquist criteria is used to equally intervals sample RFI signal $s_{r}(\tau)$, and the number of points is $N$. Then the compressed sampling number $M<N$. The discrete data which use the conventional uniform interval $\Delta T=1 / F_{s}$ to sample $s_{r}(\tau)$ is as follows.

$$
\tilde{\mathbf{s}}_{r}=\left[\begin{array}{llll}
\tilde{s}_{1} & \tilde{s}_{2} & \cdots & \tilde{s}_{N}
\end{array}\right]^{\mathbf{T}}, \quad \tilde{s}_{n}=s_{r}((n-1) \cdot \Delta T)
$$

Sampling data in frequency domain is

$$
\mathbf{s}_{r}=\left[\begin{array}{llll}
S_{1} & S_{2} & \cdots & S_{N}
\end{array}\right]^{\mathrm{T}}=\mathbf{F} \tilde{\mathbf{s}}_{r}
$$

Here $\mathbf{F}$ is the $N \times N$ dimensional Fourier transformation matrix. The RFI spectrum Sparse feature is that $\mathbf{s}_{r}$ only contains a small amount of great modulus value elements.

Observation data after compressed sampling can be expressed as follows.

$$
\mathbf{y}=\mathbf{A} \mathbf{s}_{r}+\mathbf{z}
$$

Here, compressed sampling to the "noise" signal $z(\tau)$ is

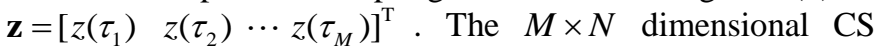
matrix in the formula above is $\mathrm{A}$.

$$
\mathbf{A}=\left[\begin{array}{cccc}
e^{j 2 \pi f_{1} \tau_{1}} & e^{j 2 \pi f_{2} \tau_{1}} & \cdots & e^{j 2 \pi f_{N} \tau_{1}} \\
e^{j 2 \pi f_{1} \tau_{2}} & e^{j 2 \pi f_{2} \tau_{2}} & \cdots & e^{j 2 \pi f_{N} \tau_{2}} \\
\vdots & \vdots & \ddots & \vdots \\
e^{j 2 \pi f_{1} \tau_{M}} & e^{j 2 \pi f_{2} \tau_{M}} & \cdots & e^{j 2 \pi f_{N} \tau_{M}}
\end{array}\right], f_{n}=(n-1) \cdot \frac{F_{s}}{N}
$$

To RFI suppression problem, observational data $\mathbf{y}$ need to be used to estimate $\mathbf{s}_{r}$ first.

\section{RFI SUPPRESSION OF COMPRESSIVE SENSING SAR}

Due to the spectrum data $\mathbf{s}_{r}$ with sparse characteristic, according to the CS theory, $\mathbf{s}_{r}$ could be reconstructed through solving constrained optimization problems. But RFI suppression does not need to reconstruct $\mathbf{s}_{r}$ completely, only through the reconstruction algorithm to get the element value of RFI frequency. Assuming that the number of RFI components (with obvious great modulus value) of $\mathbf{s}_{r}$ is $K$, their positions are $\left\{p_{1}, p_{2}, \cdots, p_{K}\right\}$. The RFI suppression can be realized through the following formula.

$$
\tilde{\mathbf{y}}=\mathbf{y}-\sum_{k=1}^{K} S_{p_{k}} \mathbf{a}_{p_{k}}
$$

Here $\mathbf{a}_{p_{k}}$ is the $p_{k}$ th column vector of CS matrix A. The RFI suppression processing flow of CS SAR imaging proposed in this paper is shown in Figure 1.

Greedy algorithm ${ }^{[6]}$ can be used to achieve the reconstruction of $K$ RFI components of $\mathbf{s}_{r}$. Such algorithm is that an optimization problem of the formula (5) changes into the following solving model.

$$
\min _{\mathbf{x}}\left\|\mathbf{y}-\mathbf{A} \mathbf{s}_{r}\right\|_{2} \quad \text { s.t. } \quad\left\|\mathbf{s}_{r}\right\|_{0}=K
$$

Greedy algorithm is mainly represented by matching pursuit (MP) algorithm and orthogonal matching pursuit (OMP) algorithm ${ }^{[7,8]}$. The OMP algorithm is used in this paper to reconstruct the RFI component. To use OMP algorithms, need to input sparsity $K$ (that is the iterations of the algorithm). But RFI component is not known in advance, so it needs to be estimated first. Taking into account that the influence of the 'noise' data $\mathbf{z}$, and the synthetic aperture time of imaging, the possibility of changing the RFI component frequency position is small. 


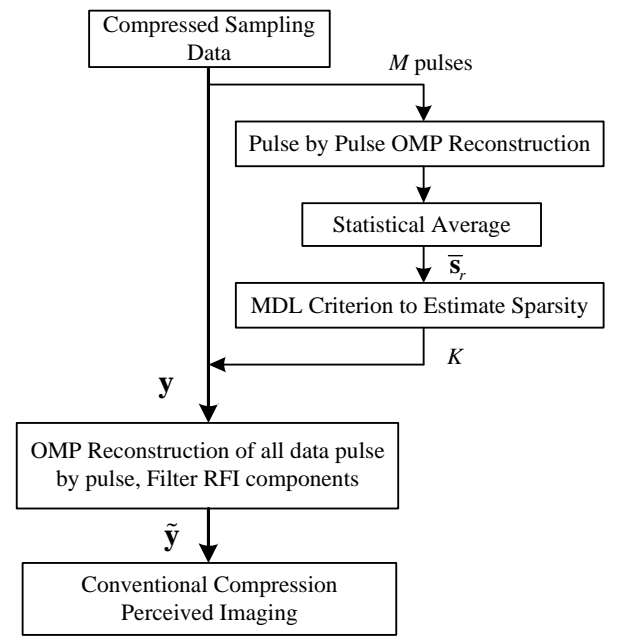

Fig.1. The RFI Suppression Processing Flow of Compressive Sensing SAR Imaging

We chose the first $M$ pulses' compression sampling data to estimate $K$. After statistical average, OMP algorithm iterations which will be used to filter RFI components by-pulse would be obtained. First of all, choose one conservative RFI frequency domain sparsity value $K_{M}$ as the OMP algorithm importing iterations. For example, put $K_{M}=N / 4$, that means the RFI component spectral range is $1 / 4$ of the signal spectrum range at the most. So, each pulse's compression sampling data $\mathbf{y}^{(m)}, m=1, \cdots, M$ can be reconstructed out their frequency domain sampling data $\hat{\mathbf{s}}_{r}^{(m)}=\left[\begin{array}{ll}\hat{S}_{1}^{(m)} & \hat{S}_{2}^{(m)} \cdots \hat{S}_{N}^{(m)}\end{array}\right]^{\mathrm{T}}$ through the OMP algorithm, its non-zero elements are the first $K_{M}$ frequency domain samples which with large modulus values. To the modulus value of each element, do the statistical average and get $\overline{\mathbf{s}}_{r}=\left[\begin{array}{lll}\bar{S}_{1} & \bar{S}_{2} \cdots \bar{S}_{N}\end{array}\right]^{\mathrm{T}}$, the value of each element is

$$
\bar{S}_{i}=\frac{1}{M} \sum_{m=1}^{M}\left|\hat{S}_{i}^{(m)}\right| \quad, \quad i=1,2, \cdots N
$$

As a result of 'noise' data's influence, the number of the non-zero element in $\overline{\mathbf{s}}_{r}$ is not $K_{M}$, but its principal components' number (the number of elements which with larger modulus value significantly) can be used as the estimated value of sparsity $K$. The number of principal components can be estimated by using MDL criterion ${ }^{[9]}$. Assuming that the number of non-zero elements of $\overline{\mathbf{s}}_{r}$ is $L$, sorting the value of element $\lambda_{l} \in\left\{\bar{S}_{1}, \bar{S}_{2}, \cdots, \bar{S}_{N}\right\}, l=1, \cdots, L$ from big to small, namely $\lambda_{1} \geq \lambda_{2} \geq \cdots \geq \lambda_{L}$. Then use the following formula to calculate after sorting.

$$
\begin{aligned}
& \operatorname{MDL}(l)=2 L(L-l) \rho(l)+\frac{1}{2} l(2 L-l) \lg (2 L) \\
& \rho(l)=\lg \left(\sum_{i=l+1}^{L} \lambda_{i}\right)-\lg (L-l)-\frac{1}{L-l} \sum_{i=l+1}^{L} \lg \lambda_{i}
\end{aligned}
$$

is the logarithm of the ratio of the arithmetic mean and the geometric mean of $K-l$ minimum component values. The estimation value of the number of the main component value is the minimum value of $\operatorname{MDL}(l)$, that is

$$
K=\min _{l} \operatorname{MDL}(l)
$$

If $K=0$, then adjudge that RFI does not exist in the compressed sampling data of the received signal, otherwise adjudge that RFI is detected.

After getting the sparsity $K$ of the frequency domain RFI components, RFI signal can be reconstructed and filtered through one by one pulse. Then enter each pulse compression sampling data $\mathbf{y}, \mathrm{CS}$ matrix $\mathbf{A}$ and iterations $K$ into the OMP algorithm to processing, get $K$ corresponding RFI component reconstruction results of $\mathbf{s}_{r}$, mark their frequency positions as $\left\{p_{1}, p_{2}, \cdots, p_{K}\right\}$. Then use formula (10) to complete the RFI signal suppression processing.

After RFI suppression, $\tilde{\mathbf{y}}$ is still compression sampling data, but not include RFI signal, the subsequent imaging processing can be done by the conventional CS SAR reconstruction algorithm ${ }^{[4,5]}$

\section{SimUlation AND VERIFICATION}

Simulation raw data used for SAR image is collected by the airborne SAR system which is working in the L-band and the center frequency is $1.25 \mathrm{GHz}$. The number of samples is $N=$ 1024. Specific system parameters are shown in Table 1. Add interference bandwidth RFI of $0.5 \mathrm{MHz}$ at a frequency of $1.265 \mathrm{GHz}$, and add single-frequency RFI signals both at $1.24 \mathrm{GHz}$ and $1.254 \mathrm{GHz}$. Set jamming-signal-noise-ratio (JSNR) at $3 \mathrm{~dB}$.

TABLE 1 L-SAR Data Parameters

\begin{tabular}{|l|l|}
\hline System Parameters & Value \\
\hline Wavelength $\lambda$ & $0.24 \mathrm{~m}$ \\
\hline LFM Signal Bandwidth $B_{r}$ & $41.255 \mathrm{MHz}$ \\
\hline Pulse Bandwidth $T_{p}$ & $16 \mu \mathrm{s}$ \\
\hline Sampling Rate $F_{s}$ & $50 \mathrm{MHz}$ \\
\hline Pulse repetition frequency PRF & $773 \mathrm{~Hz}$ \\
\hline Speed V & $118.2 \mathrm{~m} / \mathrm{s}$ \\
\hline Slant Range $R_{a}$ & $9216 \mathrm{~m}$ \\
\hline
\end{tabular}

To echo of each pulse, do the compressing sampling in a way of time domain non-uniform random sampling. Reduce $50 \%$ in the amount of data which means the compressed sampling number for each pulse echo is 512. Select the first 10 pulses of compressed sampled data to estimate the sparsity of the frequency-domain RFI components according to the flow shown in figure 1 . When OMP algorithm is used to reconstruct spectral data, input the conservative number of iterations $K_{M}=256$. After statistical average, $\overline{\mathbf{s}}_{r}$ is obtained. Then 
MDL criterion is used to obtain the estimated value of the sparsity $K=13$.

Do the RFI suppression to compression sampling data of all pulse, and do the conventional CS imaging processing according to the flow shown in figure 1. The imaging results before and after RFI suppression are shown in figure 2 . We take contrast value as the SAR image quality assessment parameter before and after suppression. The contrast is defined as the ratio of the standard deviation and the mean value of the amplitude image.

In our experiments, the image contrast of conventional CS SAR imagary processing result is 0.214 if RFI interference is not added. The image with RFI and directly CS imaging processing is shown in figure 2 (a), the contrast is 0.062 . As figure 2 (b) shows, the image after RFI suppression which is using the method proposed in this paper, the contrast is 0.202 . It can be seen that effectively suppression can be achieved to the CS SAR RFI.

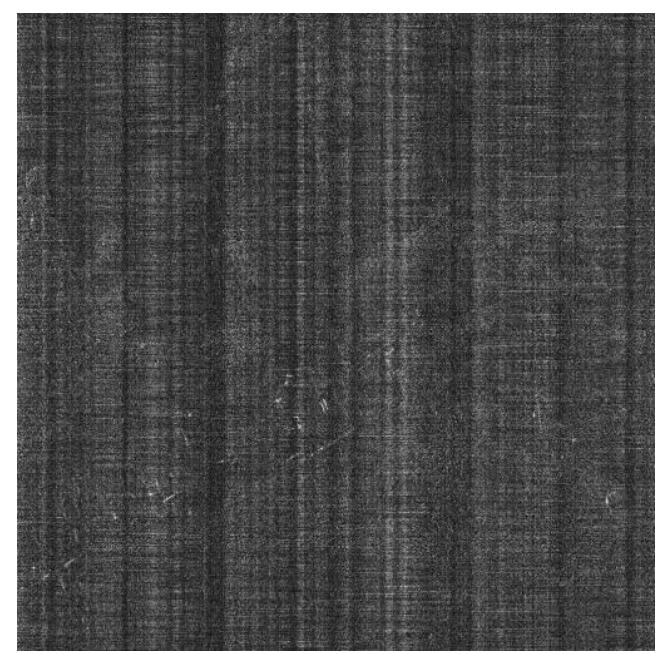

(a) Before suppressing RFI

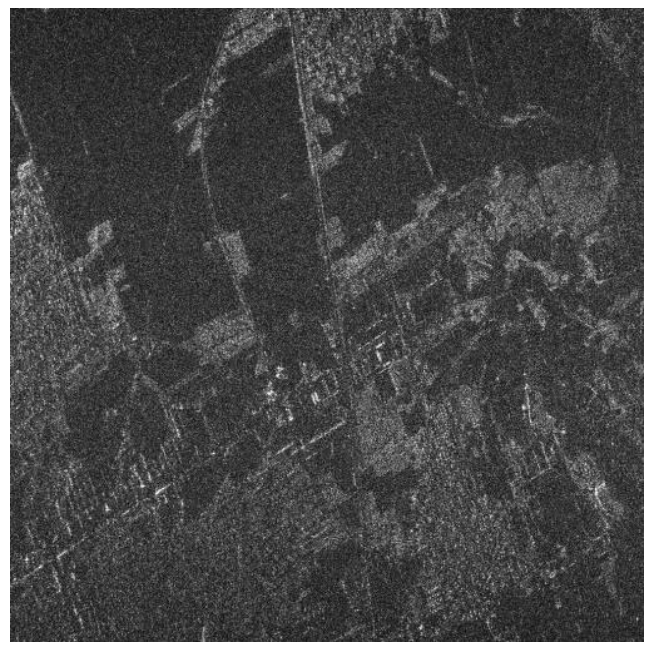

(b) After suppressing RFI

Fig.2. Suppression Effect of Compressive Sensing SAR Imaging RFI

\section{SUMMARY}

To the low-band SAR imaging system which uses CS technology, the existence of RFI would undermine the priori condition that in the sparse scene, making the subsequent reconstruction algorithm incorrectly working. For this problem, we propose a RFI suppression method for CS SAR. The method is using the OMP algorithm combined with MDL criteria to estimate the RFI components sparsity, according to the RFI sparse characteristics in the frequency domain. Then to the echo signal of each pulse, the RFI signal components are estimated and filtered in the time domain directly. Conventional CS SAR reconstruction algorithm then can be applied to achieve imaging results. This method has low computation load and restrains narrowband and broadband RFI, can be applied in the data processing of low-band CS SAR system.

\section{ACKNOWLEDGMENT}

This work was supported by the National Basic Research Program of China (2010CB731903) and in part by the National Natural Science Foundation of China (60901056, 61072112).

\section{REFERENCES}

[1] T. Miller, L. Potter, and J. McCorkle, "RFI Suppression for Ultra Wideband Radar," IEEE Trans. Aerosp. Electron. Syst., 1997, 33 (4):1142-1156.

[2] E. Candès, and M. Wakin, "An introduction to compressive sampling,” IEEE Signal Process. Mag., 2008, 25(2): 21-30.

[3] Joachim. H.G. Ender, "On compressive sensing applied to radar," Signal Processing, 2010, 90(5): 1402-1414.

[4] R. Baraniuk, and P. Steeghs, "Compressive Radar Imaging," IEEE Radar Conference, Waltham, MA, 2007: 128-133.

[5] M. Herman, and T. Strohmer, "High-resolution Radar via Compressed Sensing," IEEE Trans. Signal Processing, 2009, 57(6): 2275-2284.

[6] J. A. Tropp, "Greed is Good: Algorithmic Results for Sparse Approximation," IEEE Trans. on Info. Theory, 2004, 50(10): pp.2231-2242.

[7] Y. C. Pati, R. Rezaiifar, and P. S. Krishnaprasad, "Orthogonal Matching Pursuits: Recursive Function Approximation with Applications to Wavelet Decomposition," Proceedings of the 27th Asilomar Conference in Signals, Systems, and Computers, 1993.

[8] Tropp J. A., and Gilbert A. C, "Signal Recovery from Partial Information by Orthogonal Matching Pursuit," IEEE Transactions on Information Theory, 2007, 53 (12):4655-4666.

[9] M. Wax, and T. Kailath, "Detection of Signals by Information Theoretic Criteria," IEEE Transactions on Speech and Signal Processing, 1985, 33(2): 387-392. 